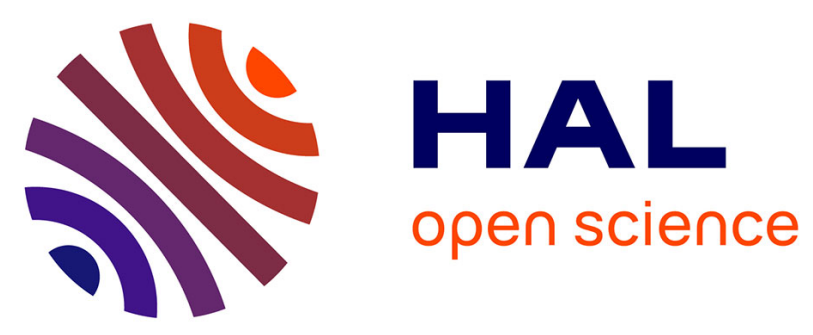

\title{
Antihypertensive Drug Regimen for High Blood Pressure Associated with Modifiable Cardiovascular Risk Factors Among Hypertensive Patients Attending Campus Teaching Hospital of Lomé, Togo, West Africa \\ Yao Potchoo, Edem Goeh-Akue, Findibe Damorou, Stéphane Lolignier, Barima Massoka, Datouda Redah, Innocent P Guissou
}

\section{To cite this version:}

Yao Potchoo, Edem Goeh-Akue, Findibe Damorou, Stéphane Lolignier, Barima Massoka, et al.. Antihypertensive Drug Regimen for High Blood Pressure Associated with Modifiable Cardiovascular Risk Factors Among Hypertensive Patients Attending Campus Teaching Hospital of Lomé, Togo, West Africa. Journal of Pharmaceutical and Pharmacological Sciences, 2018, 3 (1), pp.1-9. 10.29011/25747711. hal-03111109

\author{
HAL Id: hal-03111109 \\ https://hal.uca.fr/hal-03111109
}

Submitted on 15 Jan 2021

HAL is a multi-disciplinary open access archive for the deposit and dissemination of scientific research documents, whether they are published or not. The documents may come from teaching and research institutions in France or abroad, or from public or private research centers.
L'archive ouverte pluridisciplinaire HAL, est destinée au dépôt et à la diffusion de documents scientifiques de niveau recherche, publiés ou non, émanant des établissements d'enseignement et de recherche français ou étrangers, des laboratoires publics ou privés. 


\title{
Journal of Pharmaceutical and Pharmacological Sciences
}

\section{Antihypertensive Drug Regimen for High Blood Pressure Associated with Modifiable Cardiovascular Risk Factors Among Hypertensive Pa- tients Attending Campus Teaching Hospital of Lomé, Togo, West Africa}

\author{
Guissou $^{5}$ \\ ${ }^{1}$ Department of Pharmaceutical Sciences, University of Lomé, Togo \\ ${ }^{2}$ University of Clermont Auvergne, Neuro-Dol, BP 10448, F-63000 Clermont-Ferrand, France \\ ${ }^{3}$ Inserm, U 1107, Neuro-Dol, 63000 Clermont-Ferrand, France \\ ${ }^{4}$ University of Lomé, School of Medical Assistants, Togo \\ ${ }^{5}$ University of Ouagadougou, Laboratory of Pharmacology/ Toxicology, Burkina Faso
}

Yao Potchoo $^{1 *}$, Edem Goeh-Akue ${ }^{1}$, Findibe Damorou ${ }^{1}$, Stéphane Lolognier ${ }^{2,3}$, Barima Massoka ${ }^{4}$, Datouda Redah ${ }^{1}$, Innocent P.

"Corresponding author: Yao Potchoo, Department of Pharmaceutical Sciences, Faculty of Sciences for Health, University of Lomé, PB 1515, Lomé, Togo. Tel: +22890113478; Email: ypotchoo@hotmail.com

Citation: Potchoo Y, Goeh-Akue E, Damorou F, Lolognier S, Massoka B, et al. (2018) Antihypertensive Drug Regimen for High Blood Pressure Associated with Modifiable Cardiovascular Risk Factors Among Hypertensive Patients Attending Campus Teaching Hospital of Lomé, Togo, West Africa. J Pharma Pharma Sci: JPPS-176. DOI: 10.29011/2574-7711. 100076

Received Date: 23 October, 2018; Accepted Date: 08 November, 2018; Published Date: 16 November, 2018

\begin{abstract}
Objective: The present prospective study was aimed to target the antihypertensive drug regimen for HBP associated with modifiable cardiovascular risk factors such as stressful and sedentary lifestyle, obesity, diabetes and chronic ethylism among hypertensive patients.

Material and Method: This study was conducted among outpatients and inpatients attending the department of cardiology of Campus Teaching Hospital for hypertension conditions associated with comorbidities (stressful, sedentary lifestyle, obesity, diabetes and ethylism) and placed on antihypertensive drug therapy.

Results: Of the 112 patients treated for HBP, 43 (38.39\%) received an ambulatory treatment and 69 (61.61\%) were hospitalized. Of 43 outpatients, $37.21 \%$ and $18.60 \%$ vs $24.64 \%, 34.78 \%$ and $23.19 \%$ of 69 inpatients presented respectively 2,3 and $\geq 4$ concomitant modifiable risk factors. Regarding the antihypertensive drug regimens received and concomitant risk factor profile of patients, significant reduction of Systolic Blood Pressure (SBP) among patients with 3 risk factors and Diastolic Blood Pressure (DBP) among patients with at least, together with chronic ethylism, another risk factor among stressful, sedentary lifestyle, obesity and diabetes, was observed. In cases of complicated HBP, outpatients with 3 concomitant risk factors against inpatients with high stress, sedentary lifestyle and obesity were placed on diuretic (D), Calcium Channel Antagonist (CCA) and Angiotensin Converting Enzyme Inhibitor (ACEI) as first choice in monotherapy or in addition to Centrally Acting Antihypertensive Drug (CAAD) in suitable combinations in $2 / 3$ of total combinations prescribed.

Conclusion: antihypertensive drug regimens from mono to quadruple therapy allowed to control significantly SBP in patients with 3 concomitant modifiable risk factors and DBP among patients with at least, together with chronic ethylism, another risk factor among stress, sedentarity, obesity and diabetes. In complicated HBP with concomitant modifiable risk factors, D or CCA or ACEI or in suitable combinations in addition to CAAD were the favorite pharmacological groups prescribed.
\end{abstract}




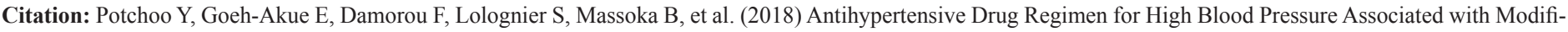

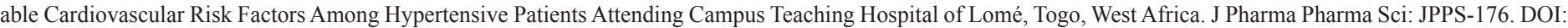
10.29011/2574-7711. 100076

Keywords: Antihypertensive Drug Regimen; Combination of Modifiable Cardiovascular Risk Factor; Hypertensive Patients; Number and type of Modifiable Cardiovascular Risk Factors; Togo

\section{Abbreviations:}

$\begin{array}{lll}\begin{array}{l}\text { ACEI } \\ \text { Inhibitor }\end{array} & : & \text { Angiotensin Converting Enzyme } \\ \text { CAAD } & : & \text { Centrally Acting Antihypertensive } \\ \text { Drug } & & \\ \text { CCA } & : & \text { Calcium Channel Antagonist } \\ \text { D } & : & \text { Diuretic } \\ \text { DBP } & : & \text { Diastolic Blood Pressure } \\ \text { ESH } & : & \text { European Society of Hypertension } \\ \text { ESC } & : & \text { European Society of Cardiology } \\ \text { HBP } & : & \text { High Blood Pressure } \\ \text { SBP } & : & \text { Systolic Blood Pressure } \\ \text { WHO } & : & \text { World Health Organization }\end{array}$

\section{Introduction}

Essential High Blood Pressure (HBP) has become a great concern in Togo in terms of prevalence, morbidity and mortality as shown by previous studies $[1,2]$. Antihypertensive drugs, in addition to an adequate dietetic regimen, are the most efficient way of managing HBP. These drugs are used either in monotherapy or most often in bi-, tri- or quadritherapy with molecules from different pharmacological groups; hence the difficulty in the choice of a better synergy and lesser risk. HBP pharmacotherapy, which requires a high level of adherence, aims at the reduction of BP cardiovascular risks (myocardial infarction, cerebra-vascular accident and stroke) [3-5] without adversely affecting the patients' quality of life.

Previous studies have targeted various modifiable cardiovascular risk factors such as HBP, high stress, sedentary lifestyle, low physical exercise, obesity or chronic ethylism [6] in the occurrence of cardiovascular diseases. To the best of our knowledge, the present prospective study, the first of its type in Togo for which very little data is available in the literature, intends to target the antihypertensive drug therapy that controlled HBP according to patient characteristics (number and modifiable risk factors profile : stressful and sedentary lifestyle, obesity, diabetes and chronic ethylism). The findings presented in this study could permit to target the suitable antihypertensive drug regimen according to HBP's conditions in association with the number, the type and combinations of such modifiable risk factors.

\section{Methods}

\section{Eligible Patients}

The criteria for enrolment was all patients having consulted or having been referred to or hospitalized in the department of cardiology of Campus Teaching Hospital. It also includes patients whose diagnosis of HBP has shown (BP higher than the limits defined by the World Health Organization (WHO)), i.e., $140 \mathrm{mmHg}$ for Systolic Blood Pressure (SBP) and $90 \mathrm{mmHg}$ for diastolic blood pressure (DBP). Patients were submitted to a check-up and then placed on antihypertensive drug therapy. Patients with irregular follow up visits, for whom collected data was incomplete, or whose adherence was poor, and those who claimed to take a traditional remedy in addition to the prescribed treatment, were excluded from the study.

\section{Material}

Blood pressure was measured using a Spengler mercury sphygmomanometer with an armband for adult of $12 \mathrm{~cm}$ large together with a Spengler stethoscope. The patients' weight was taken on a SECA scale with a maximum load of $150 \mathrm{~kg}$.

\section{Data Collection Method and Variable of Interest}

The present study is prospective, covering a period of one year. It has been conducted among the outpatients and inpatients attending the department of cardiology of Campus Teaching Hospital for hypertension conditions. A questionnaire allowed us to collect the information regarding age and gender as nonmodifiable factors, the hospitalized or ambulatory status, the BP level before and after being placed on antihypertensive therapy, the antihypertensive therapy regimens prescribed and the patients' modifiable risk factors associated to HBP such as stressful, sedentary lifestyle, obesity, diabetes and ethylism. Each modifiable factor was assessed as following:

Stressful lifestyle: Includes stressful profession and work, personality (psychological profile of type A), agitated, with domestic conflicts;

Sedentary lifestyle: Less than 30 minutes of sporty activity per day;

Obesity: Body Mass Index (BMI) more than $30 \mathrm{~g} / \mathrm{m}^{2}$;

Diabetes: Blood glucose level more than $1.1 \mathrm{~g} / \mathrm{L}$ in the morning in an empty stomach;

Chronic ethylism: Daily consumption of alcohol, more than 3 glasses of wine per day or equivalent amount for other alcoholic drinks.

The BP was measured using the auscultator method at rest, in a quiet environment. 


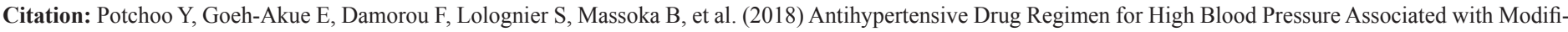

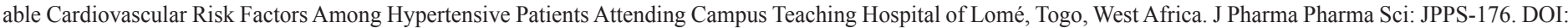
10.29011/2574-7711. 100076

The status of hypertension control was classified as controlled or uncontrolled hypertension using the limits defined previously. The mean difference between baseline SBP/DBP and that obtained after placing patients on antihypertensive therapy was calculated. These mean differences were used to compare treated groups.

\section{Study Limits}

In the modifiable cardiovascular risk factors, dietary habits, patients' lipidic profile have not been taken into consideration in the analysis of the results. The survey was not interested in the undesirable events that could occur in the course of the treatment. The size of some tested groups was limited and could not be analyzed. At the period of the study, the Ministry in charge of the Health authorized the survey. Data collection was done in the absolute anonymity.

\section{Statistical Analyses}

The treated groups according to the number and the type of modifiable cardiovascular risk factors were compared in two ways ANOVA followed by Tuckey's multiple comparisons test (Graph Pad Prism v6.07). Differences were considered significant for $\mathrm{p}<$ 0.05 .

\section{Results}

\section{Number, Type and Combinations of Modifiable Risk Factors Associated to HBP Among Ambulatory and Hospitalized Patients}

Of the 112 patients treated for HBP, 43 (38.39\%) received an ambulatory treatment and $69(61.61 \%)$ were hospitalized. Distributions of patients according to the number or combination of concomitant risk factors in a patient are presented in Table 1,2 , respectively. Among patients who normalized their BP under antihypertensive drug therapy, 37.21\%, 37.21\% and $18.60 \%$ outpatients vs $24.64 \%, 34.78 \%$ and $23.19 \%$ inpatients respectively presented cumulatively 2,3 and $\geq 4$ modifiable cardiovascular risk factors (Table 1).

\begin{tabular}{|c|c|c|c|c|}
\hline $\begin{array}{c}\text { Number of modifiable } \\
\text { cardiovascular risk } \\
\text { factors }\end{array}$ & \multicolumn{2}{|c|}{$\begin{array}{c}\text { Ambulatory } \\
\text { patients }\end{array}$} & \multicolumn{2}{c|}{$\begin{array}{c}\text { Hospitalized } \\
\text { patients }\end{array}$} \\
\cline { 2 - 5 } & $\mathbf{n 1}$ & $\mathbf{\%}$ & $\mathbf{n 2}$ & $\mathbf{\%}$ \\
\hline 0 risk factor & 2 & 4.65 & 2 & 2.90 \\
\hline 1 risk factor & 1 & 2.33 & 10 & 14.49 \\
\hline 2 risk factors & 16 & 37.21 & 17 & 24.64 \\
\hline 3 risk factors & 16 & 37.21 & 24 & 34.78 \\
\hline$\geq$ 4 risk factors & 8 & 18.60 & 16 & 23.19 \\
\hline TOTAL & $\mathbf{4 3}$ & $\mathbf{1 0 0}$ & $\mathbf{6 9}$ & $\mathbf{1 0 0}$ \\
\hline
\end{tabular}

Table 1: Distribution of patients according to the number of modifiable risk factors in addition to HBP among ambulatory patients $(\mathrm{n} 1=43)$ and hospitalized patients $(\mathrm{n} 2=69)$.

\begin{tabular}{|c|c|c|c|c|}
\hline \multirow{2}{*}{$\begin{array}{c}\text { Type of modifiable } \\
\text { cardiovascular risk factors }\end{array}$} & \multicolumn{2}{|c|}{$\begin{array}{c}\text { Ambulatory } \\
\text { patients }\end{array}$} & \multicolumn{2}{c|}{$\begin{array}{c}\text { Hospitalized } \\
\text { patients }\end{array}$} \\
\cline { 2 - 5 } & $\mathrm{n} 1$ & $\%$ & $\mathrm{n} 2$ & $\%$ \\
\hline Stress & 1 & 2.32 & 5 & 7.25 \\
\hline Stress + sedentary & 10 & 23.26 & 6 & 8.70 \\
\hline Stress + sedentary + diabetes & 2 & 4.65 & 2 & 2.90 \\
\hline Stress + sedentary + obesity & 10 & 23.26 & 9 & 13.04 \\
\hline $\begin{array}{c}\text { Chronic ethylism with another } \\
\text { risk factor among stress, } \\
\text { sedentary, obesity, diabetes }\end{array}$ & 12 & 27.91 & 32 & 46.38 \\
\hline Others & 8 & 18.60 & 15 & 21.73 \\
\hline TOTAL & $\mathbf{4 3}$ & $\mathbf{1 0 0}$ & $\mathbf{6 9}$ & $\mathbf{1 0 0}$ \\
\hline
\end{tabular}

Table 2: Distribution of patients according to the type and combinations of modifiable risk factors in addition to HBP among outpatients $(\mathrm{n} 1=43)$ and inpatients $(\mathrm{n} 2=69)$.

Outpatients with HBP associated with stressful and sedentary lifestyle were $23.26 \%$ vs $8.70 \%$ for inpatients. Life conditions including both stressful lifestyle, sedentary lifestyle and obesity in addition to HBP represented $23.26 \%$ of outpatient's vs $13.04 \%$ for inpatients. Patients with modifiable cardiovascular risk factors as at least, together with chronic ethylism, another risk factor among stressful lifestyle, sedentary lifestyle, obesity and diabetes in addition to HBP were $27.91 \%$ (outpatients) against $46.38 \%$ (inpatients) (Table 2).

\section{Blood Pressure Control Among Ambulatory and Hospitalized Patients According to The Number of Modifiable Risk Factors}

Figure 1 shows the effect of antihypertensive treatment on SBP and DBP reduction among outpatients and inpatients according to the number of concomitant modifiable risk factors. SBP reduction in patients with 3 risk factors was statistically significant ( $\mathrm{p}<0.05$; two-way ANOVA followed by Tuckey's multiple comparisons test). The reduction of BP was significantly greater in hospitalized patients compared to those who received an ambulatory treatment $(\mathrm{p}<0.0001$ for SBP; $\mathrm{p}=0.0006$ for DBP; two-way ANOVA). 


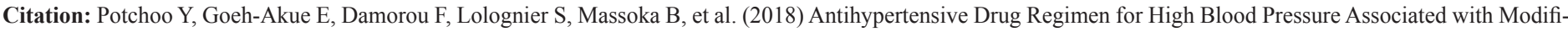

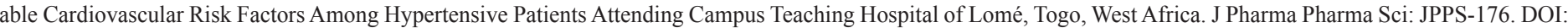
$10.29011 / 2574-7711.100076$
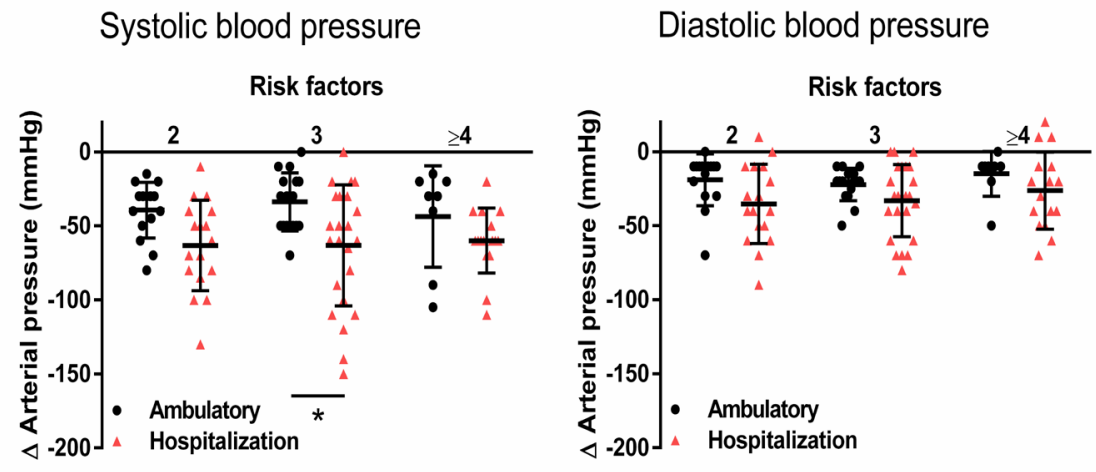

Figure 1: Effect of the number of modifiable risk factors accumulated in a subject (out-patient's vs inpatients) on the SBP and DBP reduction. *p $<0.05$; two-way ANOVA followed by Tuckey's multiple comparisons test.

\section{Blood Pressure Control Among Ambulatory and Hospitalized Patients According to The Type and Combinations of Modifiable Risk Factors}

The effect of antihypertensive treatment on SBP and DBP reduction among outpatients and inpatients according to the modifiable cardiovascular risk factors profile (type and combination) is shown in Figure 2. The findings show significant DBP reduction in patients with at least, together with chronic ethylism, another risk factor among stressful, sedentary lifestyle, obesity and diabetes ( $<0.05$; two-way ANOVA followed by Tuckey's multiple comparisons test). The reduction of BP was again significantly greater in hospitalized patients compared to those who received an ambulatory treatment ( $\mathrm{p}=0.0005$ for SBP; $\mathrm{p}=0.0077$ for DBP; two-way ANOVA).
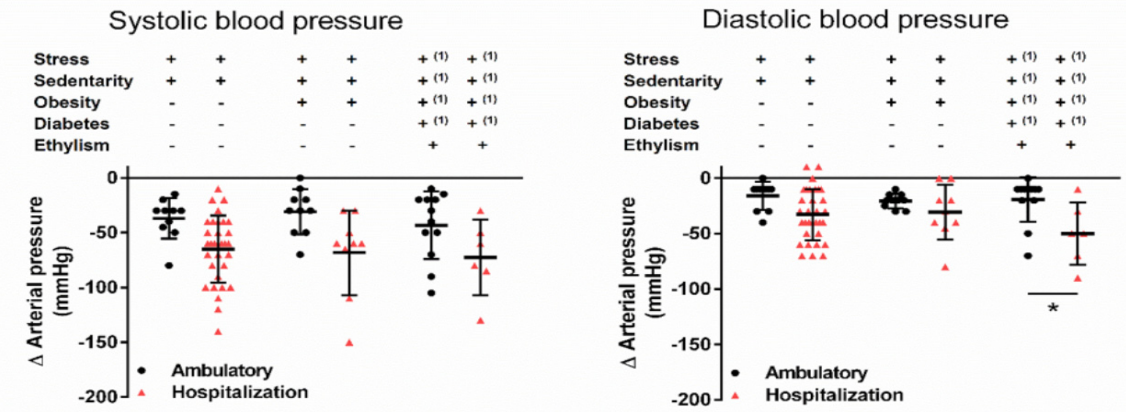

Figure 2: Effect of the type and combinations of modifiable risk factors accumulated by a subject (out or inpatients) on the SBP and DBP reduction. (1) Patients in this group present at least, together with chronic ethylism, another risk factor among stress, sedentary, obesity and diabetes. ${ }^{*} \mathrm{p}<0.05$; two-way ANOVA followed by Tuckey's multiple comparisons test.

\section{Profile of Antihypertensive Drug Regimens Prescribed Among Outpatients Compared to Inpatients According to The Number of Modifiable Risk Factors}

The following Tables 3-5 show the profile of antihypertensive drug regimens prescribed among patients suffering from HBP associated with respectively 2,3 and $\geq 4$ modifiable cardiovascular risk factors. These regimens include various pharmacological groups and range from mono to quadritherapy. Among patients with two cardiovascular risk factors in addition to HBP, physicians prescribed up to three antihypertensive drugs among outpatient's vs up to four antihypertensive drugs among inpatients. Moreover, they used bitherapy including a diuretic among ambulatory patients while hospitalized patients were placed under bitherapy with and without diuretic (CCA + CAAD or ACEI or BB + ACEI). All other combinations consisted in 3 or 4 antihypertensive drugs prescribed, including at least one diuretic. 


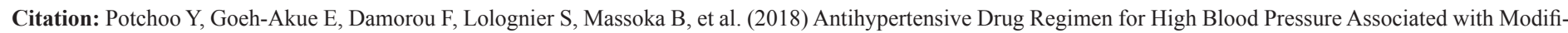

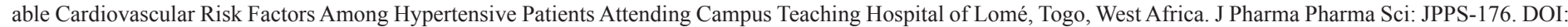
10.29011/2574-7711. 100076

\begin{tabular}{|c|c|}
\hline \multicolumn{2}{|c|}{2 risk factors } \\
\hline Ambulatory patients & Hospitalized Patients \\
\hline \multicolumn{2}{|c|}{ Monotherapy } \\
\hline $\begin{array}{l}2 \times \mathrm{CCA} \\
1 \times \mathrm{ACEI}\end{array}$ & $\begin{array}{l}1 \times \mathrm{CCA} \\
1 \times \mathrm{ACEI}\end{array}$ \\
\hline \multicolumn{2}{|c|}{ Bitherapy } \\
\hline \multirow[t]{2}{*}{$\begin{array}{c}2 \times \mathrm{D}+\mathrm{CCA} \\
3 \times \mathrm{D}+\mathrm{ACEI} \\
3 \times \mathrm{D}+\mathrm{CAAD}\end{array}$} & $\begin{array}{c}1 \times \mathrm{CCA}+\mathrm{ACEI} \\
4 \times \mathrm{D}+\mathrm{ACEI}\end{array}$ \\
\hline & $\begin{array}{c}1 \times \mathrm{BB}+\mathrm{ACEI} \\
2 \times \mathrm{CCA}+\mathrm{CAAD}\end{array}$ \\
\hline \multicolumn{2}{|c|}{ Tritherapy } \\
\hline \multirow[t]{2}{*}{$\begin{array}{c}2 \times \mathrm{D}+\mathrm{CCA}+\mathrm{ACEI} \\
1 \times \mathrm{D}+\mathrm{CCA}+\mathrm{CAAD} \\
2 \times 2 \mathrm{D}+\mathrm{CAAD} \\
\end{array}$} & $\begin{array}{c}1 \times \mathrm{D}+\mathrm{CCA}+\mathrm{ACEI} \\
1 \times \mathrm{D}+\mathrm{CCA}+\mathrm{CAAD}\end{array}$ \\
\hline & $\begin{array}{c}1 \times \mathrm{D}+\mathrm{ACEI}+\mathrm{CAAD} \\
1 \mathrm{x} \mathrm{D}+\mathrm{ACEI}+\mathrm{BB}\end{array}$ \\
\hline \multicolumn{2}{|c|}{ Quadritherapy } \\
\hline No quadritherapy regimen & $\begin{array}{c}2 \times \mathrm{D}+\mathrm{CCA}+\mathrm{ACEI}+\mathrm{CAAD} \\
1 \times \mathrm{D}+\mathrm{CCA}+\mathrm{ACEI}+\mathrm{BB}\end{array}$ \\
\hline $\begin{array}{r}\text { D: Diuretic; CCA: Calci } \\
\text { Angiotensin-Converting Enzyn } \\
\text { Antihypertensive }\end{array}$ & $\begin{array}{l}\text { lannel Antagonist; ACEI: } \\
\text { ibitor; CAAD: Centrally Acting } \\
\text { BB: Beta-Blocker }\end{array}$ \\
\hline
\end{tabular}

Table 3: Profile of antihypertensive drug regimens prescribed among ambulatory and hospitalized patients with 2 concomitant modifiable risk factors in addition to HBP.

\begin{tabular}{|c|c|}
\hline \multicolumn{2}{|c|}{3 risk factors } \\
\hline Ambulatory patients & Hospitalized patients \\
\hline \multicolumn{2}{|c|}{ Monotherapy } \\
\hline $\begin{array}{c}2 \times \mathrm{CCA} \\
1 \times \mathrm{BB} \\
1 \times \mathrm{D}\end{array}$ & No monotherapy regimen \\
\hline \multicolumn{2}{|c|}{ Bitherapy } \\
\hline $\begin{array}{c}2 \times 2 \mathrm{D} \\
5 \times \mathrm{D}+\mathrm{ACEI} \\
1 \times \mathrm{ACEI}+\mathrm{CAAD}\end{array}$ & $\begin{array}{c}2 \times \mathrm{D}+\mathrm{CCA} \\
1 \times \mathrm{CCA}+\mathrm{CAAD} \\
5 \times \mathrm{D}+\mathrm{ACEI}\end{array}$ \\
\hline \multicolumn{2}{|c|}{ Tritherapy } \\
\hline $\begin{array}{c}2 \times \mathrm{D}+\mathrm{CCA}+\mathrm{CAAD} \\
1 \times \mathrm{D}+\mathrm{ACEI}+\mathrm{CAAD} \\
1 \times 2 \mathrm{D}+\mathrm{CAAD}\end{array}$ & $\begin{array}{r}2 \times \mathrm{D}+\mathrm{CCA}+\mathrm{ACEI} \\
3 \times \mathrm{D}+\mathrm{CCA}+\mathrm{CAAD} \\
3 \times \mathrm{D}+\mathrm{ACEI}+\mathrm{CAAD} \\
1 \times \mathrm{CCA}+\mathrm{ACEI}+\mathrm{BB}\end{array}$ \\
\hline \multicolumn{2}{|c|}{ Quadritherapy } \\
\hline
\end{tabular}

\begin{tabular}{|c|c|}
\hline No quadritherapy regimen & $\begin{array}{r}4 \times \mathrm{D}+\mathrm{CCA}+\mathrm{ACEI}+\mathrm{CAAD} \\
1 \times 2 \mathrm{D}+\mathrm{CCA}+\mathrm{BB} \\
1 \times \mathrm{D}+\mathrm{CCA}+\mathrm{BB}+\mathrm{CAAD} \\
1 \times \mathrm{D}+\mathrm{ACEI}+\mathrm{BB}+\mathrm{CAAD}\end{array}$ \\
\hline $\begin{array}{c}\text { D: Diuretic; CCA: Calcium Channel Antagonist; ACEI: } \\
\text { Antiotensin-Converting Enzyme Inhibitor; CAAD: Centrally Acting } \\
\text { Antive Drug; BB: Beta-Blocker }\end{array}$ \\
\hline
\end{tabular}

Table 4: Profile of antihypertensive drug regimens prescribed among ambulatory and hospitalized patients with 3 concomitant risk factors in addition to HBP.

\begin{tabular}{|c|c|}
\hline \multicolumn{2}{|c|}{$\geq 4$ risk factors } \\
\hline Ambulatory patients & Hospitalized patients \\
\hline \multicolumn{2}{|c|}{ Monotherapy } \\
\hline $1 \times \mathrm{CCA}$ & $\begin{array}{l}1 \times C C A \\
1 \times D\end{array}$ \\
\hline \multicolumn{2}{|c|}{ Bitherapy } \\
\hline $\begin{array}{l}2 \times \mathrm{D}+\mathrm{CCA} \\
3 \times \mathrm{D}+\mathrm{ACEI}\end{array}$ & $\begin{array}{c}3 \times \mathrm{D}+\mathrm{CCA} \\
1 \times \mathrm{CCA}+\mathrm{BB} \\
1 \times \mathrm{CCA}+\mathrm{ACEI} \\
2 \times \mathrm{D}+\mathrm{CAAD} \\
\end{array}$ \\
\hline \multicolumn{2}{|c|}{ Tritherapy } \\
\hline $\begin{array}{c}1 \times \mathrm{D}+2 \mathrm{CAAD} \\
1 \times 2 \mathrm{D}+\mathrm{CCA}\end{array}$ & $\begin{array}{r}3 \times \mathrm{D}+\mathrm{CCA}+\mathrm{ACEI} \\
1 \times \mathrm{D}+\mathrm{CCA}+\mathrm{CAAD} \\
1 \times \mathrm{D}+\mathrm{ACEI}+\mathrm{CAAD}\end{array}$ \\
\hline \multicolumn{2}{|c|}{ Quadritherapy } \\
\hline No quadritherapy regimen & $\begin{array}{c}1 \times \mathrm{D}+\mathrm{CCA}+\mathrm{ACEI}+\mathrm{CAAD} \\
1 \times 2 \mathrm{D}+\mathrm{ACEI}+\mathrm{CAAD}\end{array}$ \\
\hline $\begin{array}{r}\text { D: Diuretic; CCA: Cal } \\
\text { Angiotensin-Converting Enzy } \\
\text { Antihypertensiv }\end{array}$ & $\begin{array}{l}\text { Channel Antagonist; ACEI: } \\
\text { nhibitor; CAAD: Centrally Acting } \\
\text { g; BB: Beta-Blocker }\end{array}$ \\
\hline
\end{tabular}

Table 5: Profile of antihypertensive drug regimens prescribed among ambulatory and hospitalized patients with 4 or more concomitant risk factors in addition to HBP.

Ambulatory patients with HBP associated with three modifiable cardiovascular risk factors, received monotherapy or a combination of 2 to 3 antihypertensive drugs while the hospitalized patients received combinations of 2, 3 or 4 antihypertensive drugs. Both groups of patients were placed on bitherapy without diuretic (ACEI + CAAD for outpatients vs CCA + CAAD for inpatients). Moreover, only inpatients received a tritherapy without diuretic $(\mathrm{CCA}+\mathrm{ACEI}+\mathrm{BB})$. Inpatients were placed on four different quadritherapies including a diuretic of which the most used was the combination made of D + CCA + ACEI + CAAD.

Within the patients with concomitant $\geq 4$ modifiable cardiovascular risk factors in addition to HBP, physicians 


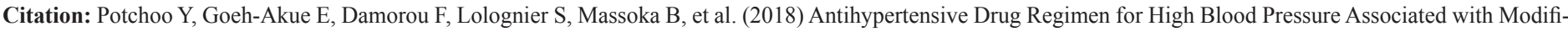

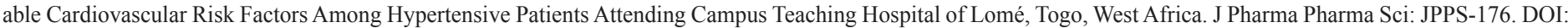
10.29011/2574-7711. 100076

prescribed mono, bi and tritherapy among outpatients, and those regimens plus quadritherapy among inpatients. Two hospitalized patients received a bitherapy without diuretic (CCA + ACEI or $\mathrm{BB})$. While outpatients were treated with a tritherapy including at least one diuretic made of $\mathrm{D}+2 \mathrm{CAAD}$ or $2 \mathrm{D}+\mathrm{CCA}$, inpatients were treated with a regimen based on tritherapies or quadritherapies with at least one diuretic but the most combinations comprised a CAAD.

\section{Profile of Antihypertensive Drug Regimens Prescribed Among Ambulatory Patients Compared to Hospitalized Patients According to Type and Combinations of Modifiable Risk Factors}

Tables 6-8 show the profile of antihypertensive drug regimens prescribed among patients suffering from HBP associated with respectively multiple combinations of modifiable risk factors. Patients with HBP associated with stressful and sendentary lifestyle as cardiovascular modifiable risk factors were placed on mono, bi and tritherapy (outpatients) against mono, bi, tri and quadritherapy (inpatients). A bitherapy without diuretic (CCA + CAAD) was prescribed for one hospitalized patient. Other combinations such as tri or quadritherapy included at least one diuretic.

\begin{tabular}{|c|c|}
\hline \multicolumn{2}{|c|}{ Stress and sedentary lifestyle } \\
\hline Ambulatory patients & Hospitalized patients \\
\hline \multicolumn{2}{|c|}{ Monotherapy } \\
\hline $1 \times$ ACEI & $1 \times \mathrm{CCA}$ \\
\hline \multicolumn{2}{|c|}{ Bitherapy } \\
\hline $\begin{array}{l}2 \times D+C C A \\
3 \times D+A C E I\end{array}$ & $\begin{array}{c}1 \times \mathrm{D}+\mathrm{ACEI} \\
1 \times \mathrm{CCA}+\mathrm{CAAD}\end{array}$ \\
\hline \multicolumn{2}{|c|}{ Tritherapy } \\
\hline $\begin{array}{c}2 \times \mathrm{D}+\mathrm{CCA}+\mathrm{ACEI} \\
2 \times 2 \mathrm{D}+\mathrm{CAAD}\end{array}$ & $\begin{array}{c}1 \times \mathrm{D}+\mathrm{CCA}+\mathrm{ACEI} \\
1 \times \mathrm{D}+\mathrm{ACEI}+\mathrm{CAAD}\end{array}$ \\
\hline \multicolumn{2}{|c|}{ Quadritherapy } \\
\hline No quadritherapy regimen & $1 \mathrm{x} \mathrm{D}+\mathrm{CCA}+\mathrm{ACEI}+\mathrm{CAAD}$ \\
\hline $\begin{array}{r}\text { D: Diuretic; CCA: Cal } \\
\text { Angiotensin-Converting Enz } \\
\text { Antihypertensiv }\end{array}$ & $\begin{array}{l}\text { Channel Antagonist; ACEI: } \\
\text { hibitor; CAAD: Centrally Acting } \\
\text { g; BB: Beta-Blocker }\end{array}$ \\
\hline
\end{tabular}

Table 6: Profile of antihypertensive drug regimens prescribed among out and inpatients with stressful and sedentary lifestyle as modifiable risk factors in addition to HBP.

\begin{tabular}{|c|c|}
\hline \multicolumn{2}{|c|}{ Stressful, sedentary lifestyle and obesity } \\
\hline Ambulatory patients & Hospitalized patients \\
\hline \multicolumn{2}{|c|}{ Monotherapy } \\
\hline $1 \times$ CCA \\
$1 \times$ BB \\
$1 \times$ D
\end{tabular}

\begin{tabular}{|c|c|}
\hline \multicolumn{2}{|c|}{ Bitherapy } \\
\hline $\begin{array}{c}1 \times 2 \mathrm{D} \\
3 \times \mathrm{D}+\mathrm{ACEI}\end{array}$ & $\begin{array}{l}3 \times \mathrm{D}+\mathrm{ACEI} \\
2 \times \mathrm{D}+\mathrm{CCA}\end{array}$ \\
\hline \multicolumn{2}{|c|}{ Tritherapy } \\
\hline $\begin{array}{l}2 \times \mathrm{D}+\mathrm{CCA}+\mathrm{CAAD} \\
1 \times \mathrm{D}+\mathrm{ACEI}+\mathrm{CAAD}\end{array}$ & $\begin{array}{l}1 \times \mathrm{D}+\mathrm{CCA}+\mathrm{CAAD} \\
1 \times \mathrm{D}+\mathrm{ACEI}+\mathrm{CAAD}\end{array}$ \\
\hline \multicolumn{2}{|c|}{ Quadritherapy } \\
\hline No quadritherapy regimen & $\begin{array}{c}1 \times \mathrm{D}+\mathrm{CCA}+\mathrm{ACEI}+\mathrm{CAAD} \\
1 \times \mathrm{D}+\mathrm{ACEI}+\mathrm{BB}+\mathrm{CAAD}\end{array}$ \\
\hline $\begin{array}{r}\text { D: Diuretic; CCA: C } \\
\text { Angiotensin-Converting En } \\
\text { Antihypertens }\end{array}$ & $\begin{array}{l}\text { Channel Antagonist; ACEI: } \\
\text { Inhibitor; CAAD: Centrally Acting } \\
\text { ug; BB: Beta-Blocker }\end{array}$ \\
\hline
\end{tabular}

Table 7: Profile of antihypertensive drug regimens prescribed among out and inpatients with concomitant high stress, sedentary lifestyle and obesity as modifiable risk factors in addition to HBP.

\begin{tabular}{|c|c|}
\hline \multicolumn{2}{|c|}{$\begin{array}{l}\text { Chronic ethylism with another risk factor among stressful, sedentary } \\
\text { lifestyle, obesity and diabetes }\end{array}$} \\
\hline Ambulatory patients & Hospitalized patients \\
\hline \multicolumn{2}{|c|}{ Monotherapy } \\
\hline $2 \times \mathrm{CCA}$ & $\begin{array}{c}1 \times \mathrm{CCA} \\
1 \times \mathrm{ACEI} \\
1 \times \mathrm{D}\end{array}$ \\
\hline \multicolumn{2}{|c|}{ Bitherapy } \\
\hline $\begin{array}{c}1 \times 2 \mathrm{D} \\
22 \times \mathrm{D}+\mathrm{CCA} \\
4 \times \mathrm{D}+\mathrm{ACEI}\end{array}$ & $\begin{array}{c}4 \times \mathrm{D}+\mathrm{CCA} \\
2 \times \mathrm{D}+\mathrm{ACEI} \\
1 \times \mathrm{CCA}+\mathrm{ACEI} \\
2 \times \mathrm{CCA}+\mathrm{CAAD}\end{array}$ \\
\hline \multicolumn{2}{|c|}{ Tritherapy } \\
\hline $\begin{array}{c}1 \times \mathrm{D}+\mathrm{CCA}+\mathrm{CAAD} \\
1 \times 2 \mathrm{D}+\mathrm{CAAD} \\
1 \times \mathrm{D}+2 \mathrm{CAAD}\end{array}$ & $\begin{array}{c}5 \times \mathrm{D}+\mathrm{CCA}+\mathrm{ACEI} \\
3 \times \mathrm{D}+\mathrm{CCA}+\mathrm{CAAD} \\
1 \times \mathrm{D}+\mathrm{ACEI}+\mathrm{CAAD} \\
1 \times \mathrm{CCA}+\mathrm{ACEI}+\mathrm{CAAD} \\
1 \times \mathrm{CCA}+\mathrm{ACEI}+\mathrm{BB}\end{array}$ \\
\hline \multicolumn{2}{|c|}{ Quadritherapy } \\
\hline No quadritherapy regimen & $\begin{array}{c}6 \times \mathrm{D}+\mathrm{CCA}+\mathrm{ACEI}+\mathrm{CAAD} \\
1 \times 2 \mathrm{D}+\mathrm{ACEI}+\mathrm{CAAD} \\
1 \times \mathrm{D}+\mathrm{CCA}+\mathrm{ACEI}+\mathrm{BB} \\
1 \times \mathrm{D}+\mathrm{CCA}+\mathrm{BB}+\mathrm{CAAD}\end{array}$ \\
\hline $\begin{array}{r}\text { D: Diuretic; CCA: Cal } \\
\text { Angiotensin-Converting Enz } \\
\text { Antihypertensiv }\end{array}$ & $\begin{array}{l}\text { Channel Antagonist; ACEI: } \\
\text { nhibitor; CAAD: Centrally Acting } \\
\text { gg; BB: Beta-Blocker }\end{array}$ \\
\hline
\end{tabular}

Table 8: Profile of antihypertensive drug regimens prescribed among out and inpatients with at least together with chronic ethylism, another risk factor among stressful, sedentary lifestyle, obesity and diabetes as modifiable risk factors in addition to HBP. 


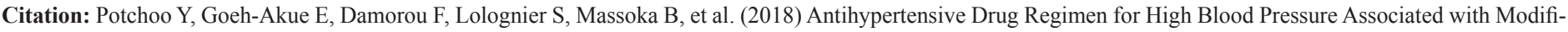

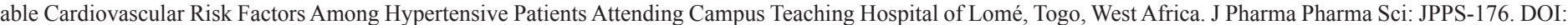
10.29011/2574-7711. 100076

The profiles of antihypertensive regimens prescribed among ambulatory patients were mono, bi and tritherapy vs bi, tri and quadritherapy among hospitalized patients, both groups suffering from stressful, sendentary lifestyle and obesity as risk factors in addition to HBP. All other combinations used included at least one diuretic. Six out of ten of them included a CAAD. Regarding modifiable cardiovascular risk factors like at least, together with chronic ethylism, another risk factor among stressful, sedentary lifestyle, obesity and diabetes, outpatients received mono, bi and tritherapy against mono, bi, tri and quadritherapy for inpatients. Moreover, among these latter, three benefited from bitherapy $(\mathrm{CCA}+\mathrm{CAAD}$ and $\mathrm{CCA}+\mathrm{ACEI})$ and two from tritherapy (CCA $+\mathrm{ACEI}+\mathrm{CAAD}$ or BB) without diuretic. Some of them received four different antihypertensive drug combinations all including at least one diuretic of which the combination of D + CCA + ACEI + CAAD was the most prescribed as in case of inpatients with tree risk factors.

\section{Discussion}

The prevalence of modifiable risk factor number in a hypertensive patient according to his statute (out or inpatient) is not available within Togolese population but considering the whole cohort with HBP, more than three out of four subjects have at least one other cardiovascular risk factor [7]. Due to the paucity of literature related to this topic (prevalence of modifiable risk factor combination among hypertensive outpatient versus inpatient) within the Togolese population, our findings could not be compared. However, considering these risk factors individually, Baragou et al. reported modifiable cardiovascular risk factors of stress $(43 \%)$, sedentary lifestyle $(41 \%)$, obesity $(25.2 \%)$, alcohol use (11\%) and diabetes (7.3\%) among adult population in an urban area of Lomé (Togo) [8] of whom our population study is issued. These are various and important risk factors to fight in order to reduce the risk for $\mathrm{HBP}$.

Concerning SBP/DBP control among patients treated with different antihypertensive regimens, SBP was significantly reduced in patients with 3 risk factors and DBP in patients with at least, together with chronic ethylism, another risk factor among stressful, sedentary lifestyle, obesity and diabetes. Excluding CAAD, our findings are in accordance with the guidelines from European Society of Hypertension (ESH)/European Society of Cardiology (ESC) who reported that D, CCA and ACEI are included among pharmacological groups recommended as suitable for initiation and maintenance of antihypertensive treatment either as monotherapy or in suitable combinations [9].

The combination made of $\mathrm{D}+\mathrm{CCA}+\mathrm{ACEI}+\mathrm{CAAD}$ is supported by the differences between the pharmacological classes with respect to target-organ damage and prevention of cardiovascular events. So the treatment should be individualized according to concomitant risk factors and diseases, and depend on age, biochemical and hemodynamic measurements [10]. Indeed, the combination including CCA + ACEI is classified as first intention combination because of its fully additive BP reduction [10-13]. Besides, the benefit of this combination is highlighted through its cardioprotective, vasculoprotective and renoprotective effects, in part mediated through a reduction in oxidative stress, a decreased interstitial fibrosis, cell migration and growth, and the prevention of endothelial dysfunction. Some antihypertensive agents may indeed produce drug-specific benefits beyond BP lowering $[14,15]$.

Among patients with HBP associated with at least, together with chronic ethylism, another risk factor among stressful, sendentary lifestyle, obesity and diabetes, the number of antihypertensive drug combinations prescribed were higher among inpatients: 13 different combinations against 6 for outpatients. This result could be explained by the severity and the complication conditions among these patients (multiple risk factor, diabetes which places hypertensive patients automatically in the highest risk category because of the enhancement of vascular and renal injury and its consequences [16-18], resistance to antihypertensive treatment) indicating that the use of a combination should be the first-line treatment.

Our study showed that prescribers resorted also to monotherapy to treat patients with multiple cardiovascular risk factors, but more among outpatients than inpatients. Besides, when that regimen is sufficient to control BP, they preferred CCA whatever the patient's status certainly because of some of its advantages (effectiveness notably in the black race patients [19], no adverse effects on lipid or carbohydrate, well tolerated notably in diabetic patients [10]). Among CCA group, lercanidipine, a lipophilic agent with a long receptor half-life [20] exhibited some advantages in various clinical studies: effective in elderly and younger patients with concomitant disease or risk factors such as obesity [21], diabetes mellitus [21,22], reduction in insulin resistance [23], across all risk levels and most effective in those with the highest risk [24]. However, Tonje et al. [10] reported that a blocker of renin angiotensin system (ACEI) should be preferred when monotherapy is sufficient.

Our study also highlights the place of CAAD (Central Adrenolytic Agents) in the management of complicated HBP (associated with multiple modifiable cardiovascular risk factors) in an African context. Indeed, outpatients with 3 risk factors and inpatients with high stress, sedentary lifestyle and obesity were placed on 2/3 combinations including CAAD. Our results show that this class of drug has its place in the management of HBP, in contrast with data from developed countries where its place is 


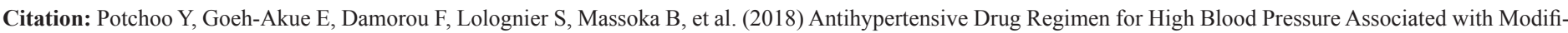

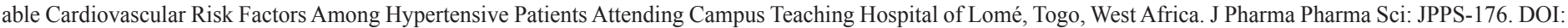
10.29011/2574-7711. 100076

reduced. In these countries, $\mathrm{BB}$, another adrenolytic agent has an important place in the management of HBP $[5,10]$ whereas their use is limited in Togolese, and even more generally in an African context. Regarding our study context, prescribers should consider the fact that BB increase the risk of developing new-onset diabetes mellitus, particularly when combined with thiazide diuretics [25]. That class of diuretic in high doses may worsen glycemic control by impairing insulin secretion and decreasing peripheral insulin sensitivity [10]. The metabolic adverse effects of BB are not seen with vasodilating noncardioselective third-generation $\mathrm{BB}$ such as carvedilol, a lipophilic and non-intrinsic sympathomimetic agent $[26,27]$ which exhibits favorable effects on metabolic parameters, eg, glycemic control, insulin sensitivity [28,29], and decrease lowdensity lipoprotein cholesterol [30,31], suggesting that it could be considered in the treatment of patients with metabolic syndrome or diabetes [32]. Finally, while the guidelines from ESH/ESC recommended $\mathrm{D}, \mathrm{BB}, \mathrm{CCA}, \mathrm{ACEI}$ and $\mathrm{ARB}$ for initiation and maintenance of antihypertensive treatment, either as monotherapy or in suitable combinations [9], the profile of suitable classes of this study includes D, CCA, ACEI as first choice in monotherapy or in addition to CAAD in suitable combinations.

\section{Conclusions}

The results of the present study indicate that antihypertensive drug regimens including mono to quadruple therapy reduced significantly SBP in patients with 3 concomitant modifiable risk factors and DBP among patients with at least, together with chronic ethylism, another risk factor among stress, sedentarity, obesity and diabetes. Regarding antihypertensive regimen profiles, monotherapy was used to treat outpatients more than inpatients. Besides, when this regimen is sufficient to control BP, prescribers preferred CCA whatever the patient's status (out or inpatient). This study also allowed us to highlight the place of CAAD in the management of complicated HBP associated with multiple modifiable cardiovascular risk factors in our context. Indeed, among outpatients with 3 risk factors against inpatients with high stress, sendentary lifestyle and obesity; D, CCA, and ACEI were prescribed as first choice antihypertensive drugs for the initiation and maintenance of antihypertensive treatment either as monotherapy or in addition to CAAD in suitable combinations in two out of tree of total combinations prescribed. Additional studies including a more important number of patients are needed to elucidate the relation between number, type and combinations of modifiable cardiovascular risk factors associated to HBP and antihypertensive drug regimens.

\section{Acknowledgements}

The authors would like to thank: The Staff of Campus Teaching Hospital for accepting the survey and the use of their facilities, and Professor Komlan ESSIZEWA for the improvement of the English language.

\section{Conflict of Interest Declaration}

The authors have no conflict of interest (patent or stock ownership, membership of a company board of directors, membership of an advisory board or committee for a company, and consultancy for or receipt of speaker's fees from a company) to declare.

\section{References}

1. Damorou F, Togbossi E, Pessinaba S, Soussou B (2008) Epidemiology and Diagnostic Circumstances of Arterial Hypertension in the Hospital Environment in Kpalime (Second Largest City in Togo). Mali Med 23: 17-20.

2. Damorou F, Pessinaba S, Tcherou T, Yayehd K, Ndassa SM, et al. (2011) Arterial Hypertension in Black Subjects over 50 Years of Age in Lomé: Epidemiological Aspects and Evaluation of Cardiovascular Risk (Prospective and Longitudinal Study of 1485 patients). Ann Cardiol Angeiol (Paris) 60: 61-66.

3. Staffileno BA (2005) Treating Hypertension with Cardioprotective Therapies, the Role of ACE Inhibitors, ARBs, and Beta-Blockers. J Cardiovasc Nurs 20: 354-364.

4. Rahn KH (2003) Recent Intervention Studies with Antihypertensive Drugs and Their Influence on Guidelines. (2003) Medi-zinische Klinik(Munich) 98: 771-775.

5. Gradman AH, Basile JN, Carter BL, Bakris GL, Materson BJ, et al. (2011) Combination Therapy in Hypertension. J Am Soc Hypertens 13: 146-154.

6. Ghannem H, Fredj AH (1997) Epidemiological transition and cardiovascular risk factors in Tunisia. Rev Epidemiol Santé Publique 45: 286-292.

7. Asmar R, Vol S, Pannier B, Brisac AM, Tichet J, et al. (2001) High blood pressure and associated cardiovascular risk factors in France. $\mathrm{J}$ Hypertens 19: 1727-1732.

8. Baragou S, Djibril M, Atta B, Damorou F, Pio M (2012) Prevalence of cardiovascular risk factors in an urban area of Togo: a WHO STEPSwise approach in Lome, Togo. Cardiovasc J Afr 23: 309-312.

9. Mancia G, De Backer G, Dominiczak A, Cifkova R, Fagard R, et al. (2007) Guidelines for the management of arterial hypertension: The Task Force for the Management of Arterial Hypertension of the European Society of Hypertension (ESH) and of the European Society of Cardiology (ESC). Eur Heart J 28: 1462-1536.

10. Aksnes TA, Skårn SN, Kjeldsen SE (2012) Treatment of Hypertension in Diabetes. What Is the Best Therapeutic Option? Expert Rev Cardiovasc Ther 10: 727-734.

11. Frishman WH, Ram CVS, McMahon FG, Chrysant SG, Graff A, et al. (1995) Comparison of amlodipine and benazepril monotherapy to amlodipine plus benazepril in patients with systemic hypertension: a randomized, double-blind, placebo controlled parallel-group study. J Clin Pharmacol 35: 1060-1066. 
Citation: Potchoo Y, Goeh-Akue E, Damorou F, Lolognier S, Massoka B, et al. (2018) Antihypertensive Drug Regimen for High Blood Pressure Associated with Modifiable Cardiovascular Risk Factors Among Hypertensive Patients Attending Campus Teaching Hospital of Lomé, Togo, West Africa. J Pharma Pharma Sci: JPPS-176. DOI: $10.29011 / 2574-7711.100076$

12. Philipp T, Smith TR, Glazer R, Wernsing M, Yen J, et al. (2007) Two multicenter, 8-week, randomized, double-blind, placebo-controlled, parallel-group studies evaluating the efficacy and tolerabilityof amlodipine and valsartan in combination and as monotherapy in adult patients with mild to moderate essential hypertension. Clin Ther 29: 563-580.

13. Chrysant SG, Melino M, Karki S, Lee J, Heyrman R (2008) The combination of olmesartan medoxomil and amlodipine besylate in controlling high blood pressure: $\mathrm{COACH}$, a randomized, double-blind, controlled, 8-week factorial efficacy and safety study. Clin Ther 30: 587-586.

14. Park JB, Schiffrin EL (2000) Effects of antihypertensive therapy on hypertensive vascular disease. Curr Hypertens Rep 2: 280-288.

15. Luis M. Ruilope, Ernesto L. Schiffrin (2001) Blood Pressure Control and Benefits of Antihypertensive Therapy. Does It Make a Difference Which Agents We Use? Hypertension, 38 (3 Pt 2): 537-542.

16. (1997) The sixth report of the Joint National Committee on prevention, detection, evaluation, and treatment of high blood pressure. Arch Intern Med 157: 2413-2446.

17. (1999) World Health Organization: International Society of Hypertension guidelines for the management of hypertension. J Hypertens 17: 151-183.

18. Haffner SM, Lehto S, Ronnemaa T, Pyorala K, Laakso M (1998) Mortality from coronary heart disease in subjects with type 2 diabetes and in no diabetic subjects with and without prior myocardial infarction. $\mathrm{N}$ Engl J Med 339: 229-234.

19. Salako LA (1993) Hypertension in Africa and Effectiveness of Its Management with Various Classes of Antihypertensive Drugs and in Different Socio-Economic and Cultural Environments. Clinical and Experimental Hypertension 15: 997-1004.

20. Herbette LG, Vecchiarelli M, Sartani A, Leonardi A (1998) Lercanidipine: short plasma half-life, long duration of action and high cholesterol tolerance. Updated molecular model to rationalize its pharmacokinetic properties. Blood Press Suppl 2: 10-17.

21. Barrios V, Navarro A, Esteras A, Luque M, Romero J, et al. (2002) Antihypertensive efficacy and tolerability of lercanidipine in daily clinical practice. The ELYPSE Study. Eficacia de Lercanidipino y su Perfil de Seguridad. Blood Press 11: 95-100.

22. Marx A, Lichtenthal A, Milbredt C, Ott R, Tegeler S, et al. (2004) Effect of anthypertensive therapy with a new third generation calcium antagonist lercanidipine on patients with concomitant diseases. J Hypertens, 22(Suppl 2): S236.
23. Sarafidis PA, Lasaridis AN, Hatzistavri L, Zebekakis PE, Tziolas I, et al. (2004) The effect of telmisartan and lercanidipine on blood pressure and insulin resistance in hypertensive patients. American Journal of Hypertension 17: 218-219.

24. Barrios V, Calderon A, Navarro A, Barrios L, Navarro-cid J, et al. (2004) Lercanidipine is an effective and well tolerated drug in essential hypertension, independently of the cardiovascular risk. The Laura study. J Hypertens 22(Suppl 2): S235.

25. Elliott WJ, Meyer PM (2007) Incident diabetes in clinical trials of antihypertensive drugs: a network meta-analysis. Lancet 369: 201-207.

26. McTavish D, Campoli-Richards D, Sorkin EM (1993) Carvedilol. A review of its pharmacodynamic and pharmacokinetic properties, and therapeutic efficacy. Drugs 45: 232-258.

27. Dickstein K, Cohen-Solal A, Filippatos G, McMurray JJ, Ponikowski P, et al. (2008) ESC guidelines for the diagnosis and treatment of acute and chronic heart failure 2008: the Task Force for the diagnosis and treatment of acute and chronic heart failure 2008 of the European Society of Cardiology. Developed in collaboration with the Heart Failure Association of the ESC (HFA) and endorsed by the European Society of Intensive Care Medicine (ESICM). Eur J Heart Fail 10: 933-989.

28. Haenni A, Lithell H (1994) Treatment with a beta-blocker with beta 2-agonism improves glucose and lipid metabolism in essential hypertension. Metabolism 43: 455-461.

29. Malminiemi K (1995) Association between serum lipids, glucose tolerance, and insulin sensitivity during 12 months of celiprolol treatment. Cardiovasc Drugs Ther 9: 295-304.

30. Bakris GL, Fonseca V, Katholi RE, McGill JB, Messerli FH, et al (2004) GEMINI Investigators. Metabolic effects of carvedilol vs metoprolol in patients with type 2 diabetes mellitus and hypertension: a randomized controlled trial. JAMA 292: 2227-2236.

31. Messerli FH, Bell DS, Fonseca V, Katholi RE, McGill JB, et al. (2007) GEMINI Investigators. Body weight changes with beta-blocker use: results from GEMINI. Am J Med 120: 610-615.

32. Carella AM, Antonucci G, Conte M, Di Pumpo M, Giancola A, et al. (2010) Antihypertensive treatment with beta-blockers in the metabolic syndrome: a review. Curr Diabetes Rev 6: 215-221. 